\title{
Operant fixation in visual preference behavior of infants
}

\section{JOHN S. WATSON, University of California, Berkeley, Calif.}

Fixation behavior in a visual choice situation was analyzed to assess whether fixation functioned tropistically or operantly in 27 infants, with a mean age of 20 weeks. The operant view was supported in analyses of spatial position learning during the first $30-s e c$ presentation and reaction to stimulus position reversal and relearning during the second presentation.

Visual discrimination capacities of infants have frequently been assessed by a visual preference technique which presents a pair of targets and compares the amount of time Ss fixate each target (e.g., Fantz, 1961; Staples, 1932; Watson, 1966). If Ss display a reliable difference in time fixating the two targets, it is concluded that the difference in the visual displays on the targets has been discriminated.

In the standard paired comparison procedure, there is a control for position bias. This control is accomplished by presenting the targets twice with their relative positions reversed on the second presentation. It is reasoned that summing the fixation times from both presentations will provide relative preference scores for the two targets which are independent of position bias because if a $S$ constantly looks at one spatial position, he will amass an equal amount of time fixating on each target. This control for position bias makes good sense if, as apparently most researchers have implicitly assumed, fixation is a respondent behavior. As a UCR, fixation during both the original and control presentation would be expected to be tropistically guided by the stimulus target which is the more "attractive" of the two.

On the other hand, if fixation is an operant behavior, the targets would function as visual reinforcers which are contingent on the specific spatial position of fixation. With one spatial position more reinforcing than the other, the response of looking in the direction of the more highly reinforcing position would be expected to increase in strength. Assuming rapid learning, since the exposure period of a pair of targets is commonly only from 30 to $60 \mathrm{sec}$, this operant view of fixation behavior would account for the differential fixation time data obtained in most visual preference studies. However, the position bias control should produce an interesting problem for operant fixation. When, as is commonly the case, the control presentation directly follows the original presentation, the operant learning view would predict a negative transfer effect to occur at least initially in the second presentation. A S differentially reinforced to look at the spatial position on the right in the first presentation ought to begin the second presentation with a dominant tendency to look to the right even though that spatial position is now occupied by the least preferred stimulus. One would expect relearning on the second presentation, but it is not clear how fast that might occur with infant Ss.

To assess the validity of the preceding operant fixation proposal, a special reanalysis was made of visual preference data obtained in a previous study (Watson, 1966). In a portion of that study, 48 infants (six Ss of each sex at approximately $8,14,20$, and 26 weeks of age) were presented a visual choice card containing two orientations, $0 \mathrm{deg}$ and $180 \mathrm{deg}$, of a schematic face drawing. A second card on which the lateral positions of the two orientations were reversed, immediately followed the first. Ss were in supine position and presentations were made from a display rack approximately 12 in. above a S's eyes. Presentations began and lasted for $30 \mathrm{sec}$ from the time the $S$ looked to the center of the display rack.

Summing the fixation times for the two presentations in the usual fashion, the composite scores indicated a significant preference for looking for the 0 deg orientation $(p<.05,1$-tailed t). These data provide, therefore, a suitable opportunity to assess the operant fixation predictions. The data are particularly suitable since in the process of providing controls against observer bias, half the $S s$ received the $0 \mathrm{deg}$ face on the right in the first presentation and the other half received it on the left initially.

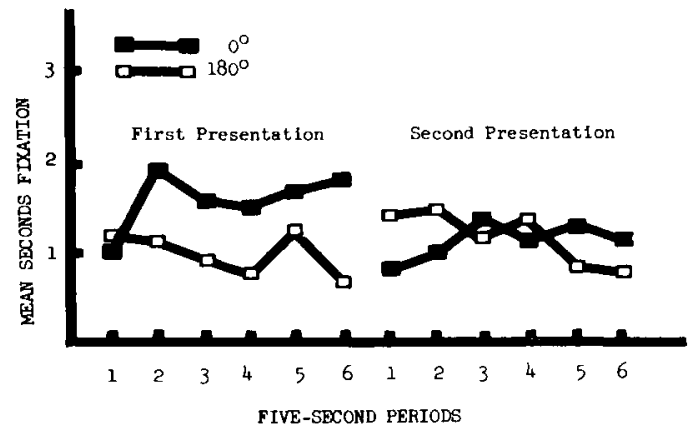

Fig. 1. Mean fixation times for the $0 \mathrm{deg}$ and $180 \mathrm{deg}$ targets per $5 \mathrm{sec}$ periods of the two visual choice presentations.

This provides an across-Ss control for position bias in both presentations.

Since the question is whether or not fixation in a visual preference situation functions as a class of operant behavior, only those Ss whose behavior provided an opportunity for differential reinforcement during the first presentation were included in the analysis. Therefore, the analysis was limited to those Ss who looked at least twice in both directions during the first $30 \mathrm{sec}$ presentation. Twenty-seven Ss met this criterion. These Ss ranged in age from 7 to 26 weeks with a mean of 20 weeks.

Data analysis involved dividing each $30-\mathrm{sec}$ presentation into six 5 -sec time periods. Thus, each $\mathrm{S}$ obtained a fixation time score for each stimulus on each of the $125-\mathrm{sec}$ periods. Figure 1 presents a graph of the mean fixation times for each stimulus across the 12 periods.

During the initial $5 \mathrm{sec}$ of the first presentation there was no difference in fixation time for the two stimuli. Yet within just the next $10 \mathrm{sec}$ a difference in fixation time on the two stimuli appeared which is significant at $p<.01$, as assessed by the Wilcoxon Matched-Pairs Signed-Ranks Test. When the relative positions of the two stimuli were reversed in the second presentation, the infants initially tended to look to the position which had previously been occupied by the 0 deg face but which was now filled by the $180 \mathrm{deg}$ face. The composite increase of fixation on the $180 \mathrm{deg}$ face and decline of fixation on the 0 deg face from the final $5 \mathrm{sec}$ of the first presentation to the initial 5 $\sec$ of the second presentation is significant at $p<.02$. This apparent reversal of stimulus preference is inexplainable from a simple tropistic view of fixation. Yet it is readily interpretable as the consequence of an acquired positional response set from the operant learning perspective. A final piece of support for the operant view comes from the fact that relearning appeared to occur during the $30 \mathrm{sec}$ of the second presentation. In this case, the composite increase of fixation on the 0 deg face and the decline on the $180 \mathrm{deg}$ face from the initial to the final $10 \mathrm{sec}$ of the second presentation is significant at $p<.01$.

While these results strongly support the theoretical view that fixation in a visual choice situation functions as an operant controlled by available visual reinforcements, at least for the age range studied here, the findings do not rule out the possibility that fixation may also function tropistically to some extent. Yet, the present data do imply that to whatever extent fixation is tropistic, this UCR function apparently can be outweighed by a very short history of differential reinforcement in visual choice situations.

The results also have a rather strong methodological implication for research using the comparison procedure for assessment of visual preference and discrimination. The standard within-S control for position bias would appear to be of dubious virtue. When presentations with reversed stimulus positions follow one another consecutively, the procedure probably increases the chance of a Type II error. Furthermore, if the two presentations 
of a specific stimuli pair were interspersed among presentations of other pairs, this procedure would probably increase Type I errors because a real effect for one pair could produce an artifactual difference for the pair presented next in the series. In sum, the present results suggest that following the presentation of two stimuli differing in relative preference value, one can expect a position preference which should not be confused with position bias.

\section{REFERENCES}

FANTZ, R. L. The origin of form perception. Scientific American, 1961, 204, 66-72.

STAPLES, R. The responses of infants to color. Journal of Experimental Psychology, 1932, 15, 119-141.

WATSON, J. S. Perception of object orientation in infants. Merrill-Palmer Quarterly, 1966, 12, 73-94. 